\title{
AROPS : THE ASIAGO RED OBJECTIVE-PRISM SURVEY
}

\author{
U. MUNARI AND R. PASSUELLO \\ Astronomical Observatories of Padova-Asiago, Italy
}

\section{Background}

AROPS is an objective-prism survey conducted at high galactic latitudes with the $67 / 92 \mathrm{~cm}(\mathrm{f}=205 \mathrm{~cm}) \mathrm{Sch}$ midt telescope of the Asiago Astrophysical Observatory (Italy). The basic technical data are:

- TP4415 high resolution plates, $20 \times 20 \mathrm{~cm}$, treated in forming gas $\left(\mathrm{H}_{2}+\mathrm{N}_{2}\right)$ and covering $24 \mathrm{deg}^{2}$ on sky

$-4^{\circ}$ UBK7 objective prism, for a dispersion of $1490 \AA \mathrm{mm}^{-1}$ at NaI D

- blue side cut-off at $4980 \AA$ using a $2 \mathrm{~mm}$ GG14 filter, for a recorded $\lambda$ range $5000-7000 \AA$

- untrailed spectra to record $V \sim 16.5$ mag stars with 40 min exposures

Two main criteria have guided the selection of the AROPS fields:

- at least 15 direct imaging plates of a given field (in any photometric band, at any exposure date since 1956) must exist in the Asiago Schmidt Plate Archive. They will help in positional measurements and will also provide information about variability and colors

- the fields must be at $|b| \geq 20^{\circ}$ and widely distributed in galactic latitude and longitude

151 fields were originally selected (for a total area of $3650 \mathrm{deg}^{2}$ ). An additional $\sim 100$ fields $\left(2400 \mathrm{deg}^{2}\right)$ have been later added on with relaxed adherence to the above selection criteria.

\section{Status Report}

High quality plates have been obtained for 153 fields (as of Aug 15, 1996), with a plate rejection rate of 1 every 4 exposed. All the plates have gone through a quality assessment which has provided:

- the accurate plate center 
- the magnitude of the faintest measurable stars

- star counts over five regions of $10^{\prime} \times 10^{\prime}$ each $\left(0.139 \mathrm{deg}^{2}\right.$ total area $)$ on fixed positions on the plate

- the evaluation of contrast, focus, PSF, background fog level and uniformity over the whole plate field

The measured mean limiting magnitude for detection of stellar continuum is $V=16$, for an average of 20,000 stars recorded on each plate.

\section{Goals}

AROPS is intended to search for, classify, catalogate and study in term of galactic distribution:

- cool stars with molecular spectra (M, S, C types)

- emission line objects of any kind

- optical counter-parts of sources from various satellite survey catalogues

\section{World Wide Web}

More information on AROPS, an updated status report and the list of fields/plates can be found on the $W e b$ at the address:

http://www.fiz.uni-lj.si/astro/alarm/arops00/arops00.html 